\title{
Ventricular flow dynamics with varying LVAD inflow cannula lengths: in-silico evaluation in a multiscale model
}

Sam Liao ${ }^{\mathrm{a}, \mathrm{b}, \mathrm{c}^{\star}}$, Michael Neidlin ${ }^{\mathrm{c}}$, Zhiyong Li ${ }^{\mathrm{a}}$, Benjamin Simpson ${ }^{\mathrm{d}}$, Shaun D. Gregory

a) Queensland University of Technology (QUT), Institute of Health and Biomedical Innovation (IHBI), Kelvin Grove, QLD 4059, Australia

b) Innovative Cardiovascular Engineering and Technology Laboratory (ICETLAB), Critical Care Research Group, The Prince Charles Hospital, Chermside, QLD 4032, Australia

c) Department of Cardiovascular Engineering, Institute of Applied Medical Engineering, Helmholtz Institute, RWTH Aachen University, Aachen 52062, Germany

d) Department of Engineering, Nottingham Trent University, Clifton Lane, Nottingham, NG11 8NS, United Kingdom

e) School of Engineering, Griffith University, Southport, QLD 4215, Australia

Key terms: Fluid-structure; Computational model; Heart Failure; Cannulation; Thrombus

\section{Abstract}

Left ventricular assist devices are associated with thromboembolic events, which are potentially caused by altered intraventricular flow. Due to patient variability, differences in apical wall thickness affects cannula insertion lengths, potentially promoting unfavourable intraventricular flow patterns which are thought to be correlated to the risk of thrombosis. This study aimed to present a 3D multiscale computational fluid dynamic model of the left ventricle (LV) developed using a commercial software, Ansys, and evaluate the risk of thrombosis with varying inflow cannula insertion lengths in a severely dilated LV. Based on a HeartWare HVAD inflow cannula, insertion lengths of 5, 19, 24 and $50 \mathrm{~mm}$ represented cases of apical hypertrophy, typical ranges of apical thicknesses and an experimental length, respectively. The risk of thrombosis was evaluated based on blood washout, residence time, instantaneous blood stagnation and a pulsatility index. By introducing fresh blood to displace pre-existing blood in the LV, after 5 cardiac cycles, $46.7 \%$, $45.7 \%, 45.1 \%$ and $41.8 \%$ of pre-existing blood remained for insertion lengths of 5 , 19, 24 and $50 \mathrm{~mm}$, respectively. Compared to the $50 \mathrm{~mm}$ insertion, blood residence time was at least $9 \%, 7 \%$ and $6 \%$ higher with the 5,19 and $24 \mathrm{~mm}$ insertion lengths, respectively. No instantaneous stagnation at the apex was observed directly after the E-wave. Pulsatility indices adjacent to the cannula increased with shorter insertion lengths. For the specific scenario studied, a longer insertion length, relative to LV size, may be advantageous to minimise thrombosis by increasing LV washout and reducing blood residence time.

\section{Introduction}

In response to worldwide shortages in donor hearts, there has been a rise in implantation of left ventricular assist devices (LVADs) as an alternative treatment to heart transplantation (Kirklin et al., 2015).

Apically implanted LVADs can develop thrombosis adjacent to the inflow cannula, at the aortic root and left ventricular outflow tract (LVOT) (Estep et al., 2010; Kurihara et al., 2011; May-Newman et al., 2013). Neurological events are the major cause of death for continuous flow LVAD patients (Kirklin et al., 2017). The occurrence of 
neurological events has been suspected to be related to the inflow cannula due to intraventricular thrombosis caused by disturbed intraventricular flow patterns

(Laumen et al., 2010; May-Newman et al., 2016; K. C. Wong et al., 2014).

Typically, only one inflow cannula design exists for each LVAD; however, patient variability in apical thicknesses alters the insertion length (Eriksson et al., 2002; Kawel et al., 2012). A longer cannula (34 mm) has shown to lower the incidence of thromboembolic events by $19 \%$ compared to a shorter cannula $(24 \mathrm{~mm})$; unfortunately, the precise cause is unknown (Schmid et al., 2008).

Many earlier computational fluid dynamic (CFD) studies lacked the recreation of physiological flow structures, owed to neglected physiological left ventricular (LV) features (Baccani et al., 2002; Khalafvand et al., 2012; Liu et al., 2012; Loerakker et al., 2008; Ong et al., 2013; Prisco et al., 2017; Tsukiya et al., 2011). Previous models have generally only recreated the LV in parts, including various degrees of LV geometry accuracy (Chnafa et al., 2016; Loerakker et al., 2008); mitral valve (MV) approximations (McCormick et al., 2014; Seo et al., 2014); and heart wall motion (Cheng et al., 2005; Doenst et al., 2009; Vedula et al., 2015; Watanabe et al., 2004). However, countless challenges are associated with developing a comprehensive model through multiscale modelling approaches, arising from the coupling of physical phenomena that cover large spatial scales (Nordsletten et al., 2011).

A notable self-developed multiscale LV model has been used to assess the effect of LVAD pulsatility but the widespread use may be limited due to accessibility (Lee et al., 2016; McCormick et al., 2014). A study on the effect of cannula designs on intraventricular flow included inherent variations in insertion lengths but the major limitation was the assumption that no heart activity was present (Liao et al., 2016). Furthermore, due to different cannula geometries, it was difficult to identify which geometrical aspect had the most significance on LV flow.

This paper aims to: 1) present a multiscale numerical model in a commercial software, Ansys 18.0 (Pennsylvania, USA), addressing the major limitation of the model used to assess inflow cannulae presented by Liao et al.; and 2) directly evaluate cannula insertion lengths with one cannula design, similar to a previous clinical study (Schmid et al., 2008).

As there are no gold standards for thrombus predictions, evaluation will be based on a combination of previously implemented metrics: LV blood residence time (BRT) (Karches and Buzas, 2011; McCormick et al., 2014), washout (Prisco et al., 2017), pulsatility index (Reider et al., 2017), and stagnation (Liao et al., 2016). BRT is the time that blood has been inside the LV since entering through the MV. This provides a relative comparison of local BRTs, identifying zones with higher thrombotic risks. Blood washout describes the rate of clearance of pre-existing blood, where "fresh" blood is introduced at the MV. This metric provides an indication of how blood flows, useful in determining "short-circuit" flow paths or areas of stasis. The pulsatility index is a normalised metric of the difference between the maximum and minimum local velocity. The idea is that if the local velocity has attenuated fluctuations, there is an 
increased risk of thrombosis. Finally, stagnation is the instantaneous blood volumes that has low velocity and strain rates, a relatively strict stasis criterion.

\section{Methods}

The multiscale numerical model consists of 3 sub-models: a CFD model, a lumped parameter network (LPN) model and a finite element model.

\subsection{Cannulated left ventricle construction}

The geometry of a severely dilated LV, end systolic volume of $460 \mathrm{ml}$, was reconstructed from computed tomography (Liao et al., 2016). A 40 and $30 \mathrm{~mm}$ diameter tube represented the left atrium and aorta, respectively, with a parametric MV placed adjacent to the aortic valve (Domenichini and Pedrizzetti, 2014; Liao et al., 2016; Mao et al., 2008). The closed MV state was a $1 \mathrm{~mm}$ plane above the MV.

The apex was used as the insertion length reference point. The cannula axis intersected the apex and MV origin. Measured from a HeartWare HVAD, the inflow cannula was $27 \mathrm{~mm}$ between the tip and sewing ring attachment. Outer and inner diameters were 20.5 and $12.6 \mathrm{~mm}$, respectively. The tip had a $3 \mathrm{~mm}$ fillet.

Four insertion lengths were investigated: based on a case of apical hypertrophic cardiomyopathy (5 mm - 15), the higher (19 mm - I19) and lower (24 mm - I24) ranges of reported apical thicknesses, and an experimental insertion length $(50 \mathrm{~mm}-$ 150). The first three cases correspond to apical thicknesses of 22, 8 and $3 \mathrm{~mm}$, respectively (Kawel et al., 2012; Lang et al., 2015).

\subsection{Computational fluid dynamics model}

\subsubsection{Meshing}

The models consisted of $\sim 1$ million cells (Ansys Meshing, Ansys 18.0, Pennsylvania, USA), refer to Figure 1. Suitable cell sizes were found through a mesh independence study (Supplementary information (SI) Section 1). No elements had a skewness above 0.9 and orthogonal quality below 0.28 indicating the mesh quality was within acceptable ranges, refer to the Ansys Meshing User Guide for metric details.

\subsubsection{Fluid model setup}

The existence of true turbulence in the LV has been of debate and many CFD studies have assumed a laminar flow regime (Arefin and Morsi, 2014; Chnafa et al., 2014; Prisco et al., 2017; Zajac et al., 2015). On average, the inlet Reynolds number was less than 1000; therefore, the flow was assumed to be laminar despite the potential for turbulence throughout the cardiac cycle. A second order implicit transient formulation was used. The simulation was solved with a Pressure Implicit with Splitting of Operator pressure-velocity coupling. Spatial discretisation for momentum and pressure was second order upwind and Pressure Staggering Option, respectively. The LV wall was a dynamic mesh zone, utilising diffusion smoothing. A 
Carreau model described the non-Newtonian blood behaviour with a density of 1060 $\mathrm{kg} / \mathrm{m}^{3}$ (Johnston et al., 2004):

$$
\eta=\eta_{\infty}+\left(\eta_{0}-\eta_{\infty}\right)\left[1+\gamma^{2} \lambda^{2}\right]^{\frac{n-1}{2}}
$$

where $\eta=$ local viscosity $\left[\frac{\mathrm{kg}}{\mathrm{ms}}\right], \gamma=$ local shear rate $\left[\frac{1}{\mathrm{~s}}\right], \eta_{\infty}=0.00345\left[\frac{\mathrm{kg}}{\mathrm{ms}}\right]$, $\eta_{0}=0.056\left[\frac{\mathrm{kg}}{\mathrm{ms}}\right], \lambda=3.313[\mathrm{~s}]$, and $n=0.3568$. The scaled convergence criteria were set at $10^{-4}$ for continuity and $x / y / z$ velocity (SI Section 2 ). The mean mass flux imbalance for was less than $6^{*} 10^{-7} \mathrm{~kg} / \mathrm{s}$.

\subsection{OD lumped parameter model}

A series of ordinary differential and algebraic equations described the cardiovascular system, represented with 10 compartments. Each vessel compartment was described using a 2-element (resistance, compliance) Windkessel model where previous work provided initial parameters (Neidlin et al., 2016). Prior to coupling, LPN tuning was performed in MATLAB (MathWorks, Natick, USA) (SI Section 3). The haemodynamic targets under LVAD support were: cardiac output $=5 \mathrm{I} / \mathrm{min}$ and mean aortic pressure between 75 and $90 \mathrm{mmHg}$, as found in Table 1. The simulated condition had an E/A ratio of 2.3, within reported ranges for LVAD patients (Estep et al., 2014). The LPN was implemented in a user-defined function (UDF) for coupling with Fluent (SI Section 3).

\subsection{Finite element model}

\subsubsection{Fictitious left ventricular wall}

Instead of applying a time-dependent Young's modulus (Cheng et al., 2005), a novel approach is presented where a constant fictitious material described the heart wall that was loaded with a time-dependent pressure. The modified material properties were as follows (Lassila et al., 2012): Young's modulus $=0.7 \mathrm{MPa}$, Poisson's ratio = 0.4 , thickness $=2 \mathrm{~mm}$ and density $=0.001 \mathrm{~kg} / \mathrm{m}^{3}$. These properties are nonphysiological and are only used to provide wall movement. The extremely low density was applied to minimise inertial effects. The wall was meshed with 2959 quadrilateral dominant cells, found after a mesh independence study where a maximum of 6596 cells resulted in a maximum deformation difference of less than $1 \%$. Maximum deformation was of interest as stroke volume was dependent on the fictitious wall.

\subsubsection{Mechanical model setup}

A pressure profile, generated from the LPN using the LV volume profile (Figure 2, top panel), was applied on the shell (Figure 2, middle panel). The maximum LV volume was correlated to $2900 \mathrm{~Pa}$ which approximately matched the LPN stroke volume. The LV volume curve was downsampled as a function of the second derivative to minimise oscillatory wall motion that results in flow instability. If the absolute value of the second derivative was less than a 0.005 , every $25^{\text {th }}$ sample was taken, else every $101^{\text {st }}$ sample. To further minimise oscillations, a dampening stiffness coefficient of 
$6.36 \mathrm{e}^{-3}$ was implemented. An example of flow instability can still be seen between 0.9-1 s of Figure 2, bottom panel. The mitral and aortic valve annuli and the cannulaLV wall interface were fixed. The ejection fraction was $10 \%$.

\subsection{OD/3D Coupling and simulation}

The multiscale model solution process can be found in Figure 3. Initialisation was performed for $11 \mathrm{~s}$ from a stationary flow field followed by simulation of 5 cardiac cycles (5 s) with $0.0002 \mathrm{~s}$ time steps. Each time step had a maximum of 25 iterations per coupling iteration with a maximum of 5 coupling iterations. The total CPU time used per model was $\sim 200$ days with an Intel Xeon $2.5 \mathrm{GHz}$ processor.

At each time step, LV wall displacement was transferred to the CFD fluid. The fluid mesh was updated and the LPN transmitral flow rate was transferred to the CFD model. During diastole, the atrium (inlet) had a mass flux inflow boundary condition. The LPN LV pressure was constantly applied at the cannula outlet. Due to differences between the CFD and LPN LV volume changes, the LPN LVAD flow rate drove the CFD inlet boundary condition. To clarify, at each time step, the LPN transmitral flow rate was initially applied at the LV inlet. After each iteration, the cannula flow rate was measured by the UDF. If the difference was less than 0.0001 $\mathrm{kg} / \mathrm{s}$ between iterations, the measured flow rate was compared with LPN LVAD flow rate and if necessary, the inlet flow was adjusted to match the LPN LVAD flow rate. When the CFD model detected that the LPN was in systole, the MV became an interior fluid and a closed MV wall was applied. In summary, the passing of information was as follows: a one-way fluid-structure interaction between the fictitious wall and LV fluid; and a one-way LPN coupling to the CFD model such that the CFD flow parameters did not influence the LPN model.

\subsection{Analysis methods}

BRT was assessed using an Eulerian approach (Ghirelli et al., 2006; Ghirelli and Leckner, 2004; Haimes, 1999; Karches and Buzas, 2011). All domains were initialised to 0 . The residence time at the inlet was $0 \mathrm{~s}$. BRT was evaluated with the volume-averaged residence time in the LV fluid domain. It must be noted that due to the Eulerian approach to assess BRT, some numerical diffusion will be present.

LV washout was assessed with virtual ink (Prisco et al., 2017). The virtual ink was transported by the resultant flow. All fluid domains were initialised with an ink concentration of 0 , with the injected ink at the inlet defined to be to 1 , representing flow of fresh blood. The rate of LV washout was calculated by the percentage of old blood in the LV, normalised by the LV volume.

A 2D pulsatility index map was defined by evaluating the difference in maximum and minimum velocities of each pixel on a plane over a cardiac cycle at $0.02 \mathrm{~s}$ intervals, normalised by the volume-averaged mean velocity over the entire cardiac cycle (Reider et al., 2017). 


$$
P I=\frac{v_{\max }-v_{\min }}{v_{\text {ave }}}
$$

Blood stagnation volume was defined by velocities of less than $0.001 \mathrm{~m} / \mathrm{s}$ with a strain rate less than $100 \mathrm{~s}^{-1}$ at which blood aggregation starts to occur (Dintenfass, 1971). The volume of interest included anything below a plane $10 \mathrm{~mm}$ superior to the apex.

Three-dimensional vortex rings were visualised using the Q-criterion (Seo et al., 2014). An isosurface threshold of $6000 \mathrm{~s}^{-1}$ was defined to track the vortex ring during early diastole. The vortex structures were not directly used to quantify the thrombotic risk but instead used to identify expected vortices to ensure recreation of physiological flow patterns (Rossini et al., 2016). The diastolic vortex rings play an important role on the resultant intraventricular flow (Bermejo et al., 2014). The LV kinetic energy was calculated by the volume-averaged dynamic pressure.

\section{Results}

All velocity fields showed a typical clockwise rotation (Figure 4). Increasing cannula lengths resulted in higher velocities around the LVOT, especially pronounced between 15 and 150 .

A characteristic vortex annulus formed during early diastole; however, a shorter insertion length disturbed the vortex by flowing into the cannula, resulting in diminished vortex structures near the septum, which were pronounced towards the end of the E-wave (Figure 5). This disturbance was accompanied by changes in peak LV kinetic energy. I5, I19, I24 and I50 had a peak kinetic energy of 207, 227, 235 and $238 \mathrm{~J} / \mathrm{m}^{3}$, respectively (SI Section 4).

The basal regions were prone to long BRTs (Figure 6). Counter-intuitively, longer insertion lengths demonstrated a longer flow path between the MV and the cannula tip. For short insertions, incoming blood travelled down towards the lateral wall and was cleared at the apex. For longer insertions, the initial flow path was similar to short insertions except at the apex, the incoming blood travelled further up the septum before being evacuated though the pump. The BRT evolution for the final 5 seconds can be found in SI Section 5. As full BRT convergence was not possible due to the computational demand required, only a trend could be concluded. Compared with 150 , the BRT was at least $9.5 \%, 7.2 \%, 5.8 \%$ longer relative to $15, \mathrm{I} 19$ and $\mathrm{I} 24$, respectively (SI Section 5).

Longer insertions resulted in faster clearance of old blood (Figure 7). During the first couple of cardiac cycles, differences in the rate of washout appeared to be insignificant; however, these minor differences compounded over time. After 5 cardiac cycles, $46.7 \%, 45.7 \%, 45.1 \%$ and $41.8 \%$ of old blood remained for 15,119 , I24 and I50, respectively. The early clearance of incoming blood was clearly evident during systole for the 15 case (Figure 6). 
A high PI was observed with the MV jet and along the septal wall (Figure 6). Low PI regions were found at the cannula-endocardium interface with the exception of I5. All cases had low PI under the posterior MV leaflet and in the central regions.

The volume of stagnation in the apical region demonstrated that there was a period, directly after the E-wave, where no volumes met the stagnation criteria (Figure 8). It can be suggested that under pulsatile conditions, perhaps specific to the simulated scenario, persistent blood stasis may not always occur at the apex and the risk of thrombosis may originate from other areas.

\section{Discussion}

The effect of inflow cannula insertion lengths on the risk of thrombosis was numerically evaluated. Results suggested that a longer cannula insertion promoted LV washout for the presented haemodynamic conditions and ventricular geometry. This finding contributes to clinical observations where patients had improved outcomes with longer inflow cannulae (Schmid et al., 2008).

Identification of low velocities around the LVOT, as similarly presented, has been suggested to facilitate thrombosis (May-Newman et al., 2013). With increasing cannula lengths, the E-wave jet circulated further to provide higher velocities around the LVOT.

No compounding effects on the rate of LV washout was found in a cannulation position study (Prisco et al., 2017). In contrast, compounding effects were observed with changes in insertion lengths. This could be attributed to the flow path taken by the incoming blood: a longer flow path from the atrium could be beneficial.

Even though insignificant differences in LV washout was reported with changes in cannula designs, minor differences indicated that longer cannula designs had faster washout, corroborating previous studies (Liao et al., 2016). Furthermore, instantaneous blood stagnation volumes were observed to fluctuate over time despite constant incoming flow into the LV, agreeing with the presented work. Despite LV geometry being the only similarity between the two simulations, it could be suggested that instantaneous stagnation cannot be the sole metric to determine the risk of thrombosis. Conceivably in other patient-specific geometries, cannulation positions/designs and heart failure conditions, persistent stasis may occur.

The cannula-endocardium interface has been commonly reported to be a region for thrombosis due to low pulsatility or stagnation (Liao et al., 2016; May-Newman et al., 2017; K. Wong et al., 2014). All cases, except for I5, had a reduced PI around the cannula, corroborating in vitro findings (May-Newman et al., 2017). The higher PI with the 15 could be attributed to lower flow disturbances leading to the apex. The PI metric can be useful in predicting where potential areas of stagnation may occur but is based on the entire cardiac cycle, thus true stagnation may not be found.

The asymmetrical diastolic vortex promoted natural blood rotation (Kheradvar et al., 2010; Pedrizzetti et al., 2010). The lower peak kinetic energy of the 15 can be 
ascribed to the increased energy dissipation by the redirection of momentum into the LVAD. Reduced vortex structures with shorter insertions has similarly been reported (Laumen et al., 2010). A longer cannula pointed towards the LVOT was suggested to have better apical and LVOT washout. It could be implied that longer cannula insertions can maintain higher flow momentum along the walls.

The optimal model resolution and approach is highly dependent on parameters of interest. Myocardium modelling may be excessive if only intraventricular flow is of concern (Watanabe et al., 2004). Modelling LVAD supported LVs with time dependent imaging may have limited versatility to assess different scenarios (Doenst et al., 2009). The time varying material property approach is highly iterative and was suggested that realistic myocardial constitutive law modelling would be of benefit (Cheng et al., 2005). Instead, the presented model simplifies the modelling process while retaining adaptability.

Naturally, there are limitations to the presented work. A single patient-specific LV and haemodynamic scenario was investigated whereby results may be specific to this study. A $50 \mathrm{~mm}$ cannula insertion may not be suitable for all patients due to the risk of flow bypassing the LV entirely. The LV was assumed to move in a balloon-like manner with internal features neglected. A laminar regime was assumed despite suggestions that intraventricular flow consists of both laminar and turbulent regimes (Chnafa et al., 2016). An on/off MV was implemented to reduce the computational demand. The assumed heart rate of $60 \mathrm{bpm}$ is low for VAD patients, thus not representing typical cases (Gupta et al., 2014; Tank et al., 2012). This results in higher flow rates per cycle, thus higher filling velocities could redirect blood further towards the LVOT. An implication is that higher-than-expected LVOT washout may be observed, in comparison to higher heart rates. As all cannulae were compared within the same condition, it is expected that the results would not dramatically change. The jump in flow rate during systole could influence the results (Figure 2 bottom panel). In comparison to a smooth flow rate profile, the difference was about $4 \%$ in relation to the volume displaced during systole. The brief period in lower flow rates through the LVAD could alter the timing that old/fresh blood is cleared; however, if sufficient local mixing has already occurred then it could be expected that no major differences would be found in regard to washout.

Future work may include investigations into different LV geometries and heart failure conditions. There are potential benefits of LVAD speed modulation, such as increasing aortic valve opening and pump washout (Holtz and Teuteberg, 2014). It is hypothesised that if aortic valve opening was promoted, superior ventricular washout could be obtained in the basal region.

\section{Conclusion}

Increasing cannula lengths decreased overall BRT and increased the rate of old blood clearance. A low pulsatility index was usually found at the cannulaendocardium interface. This work elucidated that longer BRT, especially around the LVOT, may contribute to the risk of thromboembolic events and not solely due to intermittent stagnation and low pulsatility at the apex. Inflow cannula positioning may 
benefit from locations where early clearance of incoming blood can be circumvented. Ideal insertion lengths may be relative to the LV size and cannula positioning, thus patient-specific modelling of suitable inflow cannula insertion lengths could lead to superior patient outcomes.

\section{Acknowledgements}

The authors would like to recognise the financial assistance provided by the Research Training Program, Deutscher Akademischer Austauschdienst, The Prince Charles Hospital Foundation (PRO2014-08) and the National Health and Medical Research Council Centre for Research Excellence (APP1079421). Computational resources and services used in this work were provided by the HPC and Research Support Group, Queensland University of Technology, Brisbane, Australia.

\section{Conflict of interest statementThere are no financial or personal conflicts to disclose.}

\section{References}

Arefin, M.S., Morsi, Y.S., 2014. Fluid structure interaction (FSI) simulation of the left ventricle (LV) during the early filling wave (E-wave), diastasis and atrial contraction wave (A-wave). Australas. Phys. Eng. Sci. Med. 37, 413-423. https://doi.org/10.1007/s13246-014-0250-4

Baccani, B., Domenichini, F., Pedrizzetti, G., Tonti, G., 2002. Fluid dynamics of the left ventricular filling in dilated cardiomyopathy. J. Biomech. 35, 665-671. https://doi.org/10.1016/S0021-9290(02)00005-2

Bermejo, J., Benito, Y., Alhama, M., Yotti, R., Martinez-Legazpi, P., del Villar, C.P., Perez-David, E., Gonzalez-Mansilla, A., Santa-Marta, C., Barrio, A., FernandezAviles, F., del Alamo, J.C., 2014. Intraventricular vortex properties in nonischemic dilated cardiomyopathy. AJP Hear. Circ. Physiol. 306, H718-H729. https://doi.org/10.1152/ajpheart.00697.2013

Cheng, Y., Oertel, H., Schenkel, T., 2005. Fluid-structure coupled CFD simulation of the left ventricular flow during filling phase. Ann. Biomed. Eng. 33, 567-576. https://doi.org/10.1007/s10439-005-4388-9

Chnafa, C., Mendez, S., Nicoud, F., 2016. Image-Based Simulations Show Important Flow Fluctuations in a Normal Left Ventricle: What Could be the Implications? Ann. Biomed. Eng. 1-13. https://doi.org/10.1007/s10439-016-1614-6

Chnafa, C., Mendez, S., Nicoud, F., 2014. Image-based large-eddy simulation in a realistic left heart. Comput. Fluids 94, 173-187.

https://doi.org/10.1016/j.compfluid.2014.01.030

Dintenfass, L., 1971. Viscosity and Clotting of Blood in Venous Thrombosis and Coronary Occlusions. Circ. Res. XIII, 437-445. https://doi.org/10.1161/01.RES.29.5.437

Doenst, T., Spiegel, K., Reik, M., Markl, M., Hennig, J., Nitzsche, S., Beyersdorf, F., 
Oertel, H., 2009. Fluid-Dynamic Modeling of the Human Left Ventricle:

Methodology and Application to Surgical Ventricular Reconstruction. Ann.

Thorac. Surg. 87, 1187-1195. https://doi.org/10.1016/j.athoracsur.2009.01.036

Domenichini, F., Pedrizzetti, G., 2014. Asymptotic model of fluid-tissue interaction for mitral valve dynamics. Cardiovasc. Eng. Technol. 6, 95-104.

https://doi.org/10.1007/s13239-014-0201-y

Eriksson, M.J., Sonnenberg, B., Woo, A., Rakowski, P., Parker, T.G., Wigle, E.D., Rakowski, H., 2002. Long-term outcome in patients with apical hypertrophic cardiomyopathy. J. Am. Coll. Cardiol. 39, 638-645.

https://doi.org/10.1016/S0735-1097(01)01778-8

Estep, J.D., Stainback, R.F., Little, S.H., Torre, G., Zoghbi, W. a, 2010. The role of echocardiography and other imaging modalities in patients with left ventricular assist devices. JACC. Cardiovasc. Imaging 3, 1049-1064. https://doi.org/10.1016/j.jcmg.2010.07.012

Estep, J.D., Vivo, R.P., Krim, S.R., Cordero-Reyes, A.M., Elias, B., Loebe, M., Bruckner, B.A., Bhimaraj, A., Trachtenberg, B.H., Ashrith, G., Torre-Amione, G., Nagueh, S.F., 2014. Echocardiographic evaluation of hemodynamics in patients with systolic heart failure supported by a continuous-flow LVAD. J. Am. Coll. Cardiol. 64, 1231-1241. https://doi.org/10.1016/j.jacc.2014.06.1188

Ghirelli, F., Hermansson, S., Thunman, H., Leckner, B., 2006. Reactor residence time analysis with CFD. Prog. Comput. Fluid Dyn. an Int. J. 6, 241-247. https://doi.org/10.1504/PCFD.2006.010033

Ghirelli, F., Leckner, B., 2004. Transport equation for the local residence time of a fluid. Chem. Eng. Sci. 59, 513-523. https://doi.org/10.1016/j.ces.2003.10.013

Gupta, S., Woldendorp, K., Muthiah, K., Robson, D., Prichard, R., Macdonald, P.S., Keogh, A.M., Kotlyar, E., Jabbour, A., Dhital, K., Granger, E., Spratt, P., Jansz, P., Hayward, C.S., 2014. Normalisation of haemodynamics in patients with endstage heart failure with continuous-flow left ventricular assist device therapy. Hear. Lung Circ. 23, 963-969. https://doi.org/10.1016/j.hlc.2014.04.259

Haimes, R., 1999. Using Residence Time for the Extraction of Recirculation Regions, in: 14th Computational Fluid Dynamics Conference. pp. 1-10. https://doi.org/doi.org/10.2514/6.1999-3291

Holtz, J., Teuteberg, J., 2014. Management of aortic insufficiency in the continuous flow left ventricular assist device population. Curr. Heart Fail. Rep. 11, 103-110. https://doi.org/10.1007/s11897-013-0172-6

Johnston, B.M., Johnston, P.R., Corney, S., Kilpatrick, D., 2004. Non-Newtonian blood flow in human right coronary arteries: steady state simulations. J. Biomech. 37, 709-720. https://doi.org/10.1016/j.jbiomech.2003.09.016

Karches, T., Buzas, K., 2011. Methodology to determine residence time distribution and small scale phenomena in settling tanks. WIT Trans. Eng. Sci. 70, 117-125. https://doi.org/10.2495/MPF1100101 
Kawel, N., Turkbey, E.B., Carr, J.J., Eng, J., Gomes, A.S., Hundley, W.G., Johnson, C., Masri, S.C., Prince, M.R., van der Geest, R.J., Lima, J.A.C.C., Bluemke, D.A., 2012. Normal Left Ventricular Myocardial Thickness for Middle-Aged and Older Subjects With Steady-State Free Precession Cardiac Magnetic Resonance Clinical Perspective. Circ. Cardiovasc. Imaging 5, 500-508. https://doi.org/10.1161/CIRCIMAGING.112.973560

Khalafvand, S.S., Ng, E.Y.K., Zhong, L., Hung, T.K., 2012. Fluid-dynamics modelling of the human left ventricle with dynamic mesh for normal and myocardial infarction: Preliminary study. Comput. Biol. Med. 42, 863-870. https://doi.org/10.1016/j.compbiomed.2012.06.010

Kheradvar, A., Houle, H., Pedrizzetti, G., Tonti, G., Belcik, T., Ashraf, M., Lindner, J.R., Gharib, M., Sahn, D., 2010. Echocardiographic particle image velocimetry: a novel technique for quantification of left ventricular blood vorticity pattern. J. Am. Soc. Echocardiogr. 23, 86-94. https://doi.org/10.1016/j.echo.2009.09.007

Kirklin, J.K., Naftel, D.C., Pagani, F.D., Kormos, R.L., Stevenson, L.W., Blume, E.D., Myers, S.L., Miller, M.A., Baldwin, J.T., Young, J.B., 2015. Seventh INTERMACS annual report: 15,000 patients and counting. J. Hear. Lung Transplant. 34, 1495-1504. https://doi.org/10.1016/j.healun.2015.10.003

Kirklin, J.K., Pagani, F.D., Kormos, R.L., Stevenson, L.W., Blume, E.D., Myers, S.L., Miller, M.A., Baldwin, J.T., Young, J.B., Naftel, D.C., 2017. Eighth annual INTERMACS report: Special focus on framing the impact of adverse events. J. Hear. Lung Transplant. 36, 1080-1086. https://doi.org/10.1016/j.healun.2017.07.005

Kurihara, C., Ono, M., Nishimura, T., Saito, A., Taketani, T., Hisagi, M., Nawata, K., Kinoshita, O., Morota, T., Motomura, N., Kyo, S., 2011. Use of DuraHeart® support for more than 1 year as the first successful bridge to heart transplantation in Japan. J. Artif. Organs 14, 67-69. https://doi.org/10.1007/s10047-010-0524-x

Lang, R.M., Badano, L.P., Mor-Avi, V., Afilalo, J., Armstrong, A., Ernande, L., Flachskampf, F.A., Foster, E., Goldstein, S.A., Kuznetsova, T., Lancellotti, P., Muraru, D., Picard, M.H., Rietzschel, E.R., Rudski, L., Spencer, K.T., Tsang, W., Voigt, J.U., 2015. Recommendations for cardiac chamber quantification by echocardiography in adults: An update from the American society of echocardiography and the European association of cardiovascular imaging. Eur. Heart J. Cardiovasc. Imaging 16, 233-271. https://doi.org/10.1093/ehjci/jev014

Lassila, T., Malossi, A.C.I., Stevanella, M., Votta, E., Redaelli, A., Deparis, S., 2012. Multiscale fluid-structure interaction simulation of patient-specific left ventricle fluid dynamics with fictitious elastic structure regularization. Int J Numer Methods Biomed Eng 00 1-23.

Laumen, M., Kaufmann, T., Timms, D., Schlanstein, P., Jansen, S., Gregory, S., Wong, K.C., Schmitz-Rode, T., Steinseifer, U., 2010. Flow analysis of ventricular assist device inflow and outflow cannula positioning using a naturally shaped ventricle and aortic branch. Artif. Organs 34, 798-806. 
https://doi.org/10.1111/j.1525-1594.2010.01098.x

Lee, J., Cookson, A., Roy, I., Kerfoot, E., Asner, L., Vigueras, G., 2016. Multiphysics computational modeling in CHeart 38, 150-178.

Liao, S., Simpson, B., Neidlin, M., Kaufmann, T.A.S., Li, Z., Woodruff, M.A., Gregory, S.D., 2016. Numerical prediction of thrombus risk in an anatomically dilated left ventricle: the effect of inflow cannula designs. Biomed. Eng. Online 15, 587-604. https://doi.org/10.1186/s12938-016-0262-2

Liu, G.M., Chen, H.B., Luo, F.L., Zhang, Y., Sun, H.S., Zhou, J.Y., Hu, S.S., 2012. Numerical simulation of LVAD inflow cannulas with different tip. Int. J. Chem. Eng. 2012. https://doi.org/10.1155/2012/596960

Loerakker, S., Cox, L.G.E., van Heijst, G.J.F., de Mol, B. a J.M., van de Vosse, F.N., 2008. Influence of dilated cardiomyopathy and a left ventricular assist device on vortex dynamics in the left ventricle. Comput. Methods Biomech. Biomed. Engin. 11, 649-660. https://doi.org/10.1080/10255840802469379

Mao, S.S., Ahmadi, N., Shah, B., Beckmann, D., Chen, A., Ngo, L., Flores, F.R., Gao, Y.L., Budoff, M.J., 2008. Normal thoracic aorta diameter on cardiac computed tomography in healthy asymptomatic adults: impact of age and gender. Acad. Radiol. 15, 827-34. https://doi.org/10.1016/j.acra.2008.02.001

May-Newman, K., Moon, J., Ramesh, V., Montes, R., Campos, J., Herold, B., Isingoma, P., Motomura, T., Benkowski, R., 2017. The effect of inflow cannula length on the intraventricular flow field of the EvaHeart LVAD-assisted heart. ASAIO J. 63, 592-603. https://doi.org/10.1097/MAT.0000000000000559

May-Newman, K., Vu, V., Herold, B., 2016. Modeling the Link between Left Ventricular Flow and Thromboembolic Risk Using Lagrangian Coherent Structures. Fluids 1, 38. https://doi.org/10.3390/fluids 1040038

May-Newman, K., Wong, Y.K., Adamson, R., Hoagland, P., Vu, V., Dembitsky, W., 2013. Thromboembolism is linked to intraventricular flow stasis in a patient supported with a left ventricle assist device. ASAIO J. 59, 452-5. https://doi.org/10.1097/MAT.0b013e318299fced

McCormick, M., Nordsletten, D., Lamata, P., Smith, N.P., 2014. Computational analysis of the importance of flow synchrony for cardiac ventricular assist devices. Comput. Biol. Med. 49, 83-94. https://doi.org/10.1016/j.compbiomed.2014.03.013

Neidlin, M., Corsini, C., Sonntag, S.J., Schulte-Eistrup, S., Schmitz-Rode, T., Steinseifer, U., Pennati, G., Kaufmann, T.A.S., 2016. Hemodynamic analysis of outflow grafting positions of a ventricular assist device using closed-loop multiscale CFD simulations: Preliminary results. J. Biomech. 49, 2718-2725. https://doi.org/10.1016/j.jbiomech.2016.06.003

Nordsletten, D.A., Niederer, S.A., Nash, M.P., Hunter, P.J., Smith, N.P., 2011. Coupling multi-physics models to cardiac mechanics. Prog. Biophys. Mol. Biol. 104, 77-88. https://doi.org/10.1016/j.pbiomolbio.2009.11.001 
Ong, C., Dokos, S., Chan, B., Lim, E., Al Abed, A., Bin Abu Osman, N.A., Kadiman, S., Lovell, N.H., 2013. Numerical investigation of the effect of cannula placement on thrombosis. Theor. Biol. Med. Model. 10, 35. https://doi.org/10.1186/17424682-10-35

Pedrizzetti, G., Domenichini, F., Tonti, G., 2010. On the left ventricular vortex reversal after mitral valve replacement. Ann. Biomed. Eng. 38, 769-773. https://doi.org/10.1007/s10439-010-9928-2

Prisco, A.R., Aliseda, A., Beckman, J.A., Mokadam, N.A., Mahr, C., Garcia, G.J.M., 2017. Impact of LVAD Implantation Site on Ventricular Blood Stagnation. ASAIO J. 63, 392-400. https://doi.org/10.1097/MAT.0000000000000503

Reider, C., Moon, J., Ramesh, V., Montes, R., Campos, J., Herold, B., Lorenzo, P.M., Carlos, J., Dembitsky, W., Martinez-Legazpi, P., Rossini, L., Del Alamo, J.C., Dembitsky, W., May-Newman, K., 2017. Intraventricular thrombus formation in the LVAD-assisted heart studied in a mock circulatory loop. Meccanica 52, 1-14. https://doi.org/10.1007/s11012-016-0433-z

Rossini, L., Martinez-Legazpi, P., Vu, V., Fernández-Friera, L., Pérez del Villar, C., Rodríguez-López, S., Benito, Y., Borja, M.-G., Pastor-Escuredo, D., Yotti, R., Ledesma-Carbayo, M.J., Kahn, A.M., Ibáñez, B., Fernández-Avilés, F., MayNewman, K., Bermejo, J., del Álamo, J.C., 2016. A clinical method for mapping and quantifying blood stasis in the left ventricle. J. Biomech. 49, 2152-2161. https://doi.org/10.1016/j.jbiomech.2015.11.049

Schmid, C., Jurmann, M., Birnbaum, D., Colombo, T., Falk, V., Feltrin, G., Garatti, A., Genoni, M., Gerosa, G., Göttel, P., Gummert, J., Halfmann, R., Hammel, D., Hennig, E., Kaufmann, F., Lanfranconi, M., Meyns, B., Mohr, F., Müller, J., Nikolov, D., Rucinskas, K., Scheld, H.H., Schmid, F.X., Schneider, M., Sirvydis, V., Tandler, R., Vitali, E., Vlasselaers, D., Weyand, M., Wilhelm, M., Hetzer, R., 2008. Influence of inflow cannula length in axial-flow pumps on neurologic adverse event rate: results from a multi-center analysis. J. Hear. Lung Transplant. 27, 253-260. https://doi.org/10.1016/j.healun.2007.12.007

Seo, J.H., Vedula, V., Abraham, T., Lardo, A.C., Dawoud, F., Luo, H., Mittal, R., 2014. Effect of the mitral valve on diastolic flow patterns. Phys. Fluids 26, 121901. https://doi.org/10.1063/1.4904094

Tank, J., Heusser, K., Malehsa, D., Hegemann, K., Haufe, S., Brinkmann, J., Tegtbur, U., Diedrich, A., Bara, C., Jordan, J., Strüber, M., 2012. Patients with continuous-flow left ventricular assist devices provide insight in human baroreflex physiology. Hypertension 60, 849-855. https://doi.org/10.1161/HYPERTENSIONAHA.112.198630

Tsukiya, T., Toda, K., Sumikura, H., Takewa, Y., Watanabe, F., Taenaka, Y., Tatsumi, E., 2011. Computational fluid dynamic analysis of the flow field in the newly developed inflow cannula for a bridge-to-decision mechanical circulatory support. J. Artif. Organs 14, 381-384. https://doi.org/10.1007/s10047-011-0599Z 
Vedula, V., Seo, J.-H., Lardo, A.C., Mittal, R., 2015. Effect of trabeculae and papillary muscles on the hemodynamics of the left ventricle. Theor. Comput. Fluid Dyn. https://doi.org/10.1007/s00162-015-0349-6

Watanabe, H., Sugiura, S., Kafuku, H., Hisada, T., 2004. Multiphysics simulation of left ventricular filling dynamics using fluid-structure interaction finite element method. Biophys. J. 87, 2074-2085. https://doi.org/10.1529/biophysj.103.035840

Wong, K., Samaroo, G., Ling, I., Dembitsky, W., Adamson, R., Del Álamo, J.C., MayNewman, K., 2014. Intraventricular flow patterns and stasis in the LVAD-assisted heart. J. Biomech. 47, 1485-1494. https://doi.org/10.1016/j.jbiomech.2013.12.031

Wong, K.C., Büsen, M., Benzinger, C., Gäng, R., Bezema, M., Greatrex, N., SchmitzRode, T., Steinseifer, U., 2014. Effect of inflow cannula tip design on potential parameters of blood compatibility and thrombosis. Artif. Organs 38, 810-817. https://doi.org/10.1111/aor.12369

Zajac, J., Eriksson, J., Dyverfeldt, P., Bolger, A.F., Ebbers, T., Carlhäll, C.J., 2015. Turbulent kinetic energy in normal and myopathic left ventricles. J. Magn. Reson. Imaging 41, 1021-1029. https://doi.org/10.1002/jmri.24633

\section{Table captions}

Table 1: Lumped parameter network validation. $\mathrm{MCL}=$ mock circulation loop, $\mathrm{LPN}=$ lumped parameter network.

\section{Figure captions}

Figure 1: Meshed cannulated LV models. I5, I19, I24 and I50 corresponds to insertion lengths of $5 \mathrm{~mm}, 19 \mathrm{~mm}, 24 \mathrm{~mm}$ and $50 \mathrm{~mm}$. Scale bar $=60 \mathrm{~mm}$.

Figure 2: Overview of input and output information from the models. Top panel: a comparison of the ideal stroke volume from the LPN compared to the CFD stroke volume. The LPN volume profile curve was converted to a pressure profile to provide ventricular motion. Middle panel: the converted LV volume profile to pressure with downsampled and shifted data points. Bottom panel: comparison of the ideal flow rates obtained from the LPN and flow rates in the CFD model.

Figure 3: A flow chart to visualise the passing of information and solution process of the multiscale numerical model for any given time step.

Figure 4: LV velocity profiles of a cardiac cycle on a coronal plane. 15, I19, I24 and 150 corresponds to insertion lengths of $5 \mathrm{~mm}, 19 \mathrm{~mm}, 24 \mathrm{~mm}$ and $50 \mathrm{~mm}$. Scale bar $=50 \mathrm{~mm}$.

Figure 5: Example of vortex development differences during early diastole, visualised with isosurfaces with a Q-criterion threshold of $6000 \mathrm{~s}^{-1}$. Isosurfaces are coloured with axial velocity (apex to base $=$ positive velocity) to conceptualise the rotational direction. 15 and 150 corresponds to insertion lengths of $5 \mathrm{~mm}$ and $50 \mathrm{~mm}$. Scale bar $=50 \mathrm{~mm}$. 
Figure 6: First two columns: contours of blood residence time after $11 \mathrm{~s}$ of flow initialisation and $5 \mathrm{~s}$ of simulation. It must be noted the contours of blood residence time is not absolute, due to the unfeasible computational time required to reach steady state, and is to be used only as a comparison between the presented cases. Third column: visualisation of blood washout for different inflow cannula insertion lengths. Fresh incoming blood is indicated in white while pre-existing/old blood is indicated in black. Fourth column: a pulsatility index map of the last cardiac cycle, calculated by the difference in local maximum and minimum velocities and normalised by the average velocity of the entire LV over the cardiac cycle.

Figure 7: Percentage of old blood remaining in the LV over 5 cardiac cycles, indicating differences in the rate of washout based on inflow cannula insertion lengths. I5, I19, I24 and I50 corresponds to insertion lengths of $5 \mathrm{~mm}, 19 \mathrm{~mm}, 24 \mathrm{~mm}$ and $50 \mathrm{~mm}$.

Figure 8: Mean stagnation volumes over 2 cardiac cycles, based on instantaneous velocities $<0.001 \mathrm{~m} / \mathrm{s}$ and strain rate $<100 \mathrm{~s}^{-1}$, in a region $10 \mathrm{~mm}$ above the apex. No stagnation volumes were found directly after the E-wave. The purple, blue, green and orange shaded areas corresponds to the E-wave, mid-diastole, A-wave and systole, respectively. $15, \mathrm{I19}, \mathrm{I} 24$ and 150 corresponds to insertion lengths of $5 \mathrm{~mm}$, $19 \mathrm{~mm}, 24 \mathrm{~mm}$ and $50 \mathrm{~mm}$. 
Table 1

\begin{tabular}{r|c} 
Heart Rate $(\mathrm{bpm})$ & $\begin{array}{c}\text { Restored } \\
\text { Haemodynamics }\end{array}$ \\
\cline { 2 - 2 } HeartWare HVAD Speed $(\mathrm{rpm})$ & 60 \\
Mean Aortic Pressure $(\mathrm{mmHg})$ & 2600 \\
Mean Left Atrial Pressure $(\mathrm{mmHg})$ & 83 \\
Mean Right Atrial Pressure $(\mathrm{mmHg})$ & 8 \\
Cardiac Output $(\mathrm{l} / \mathrm{min})$ & 7 \\
Mean Pulmonary Artery Pressure $(\mathrm{mmHg})$ & 18.1 \\
Systemic Vascular Resistance $\left(\right.$ dyne.s $\left./ \mathrm{cm}^{5}\right)$ & 1200 \\
Pulmonary Vascular Resistance $\left(\right.$ dyne.s $\left./ \mathrm{cm}^{5}\right)$ & 150
\end{tabular}

Journal of Biomechanics (Accepted: $1^{\text {st }}$ March 2018) 
13 Figures

Figure 1



Journal of Biomechanics (Accepted: $1^{\text {st }}$ March 2018) 
Figure 2
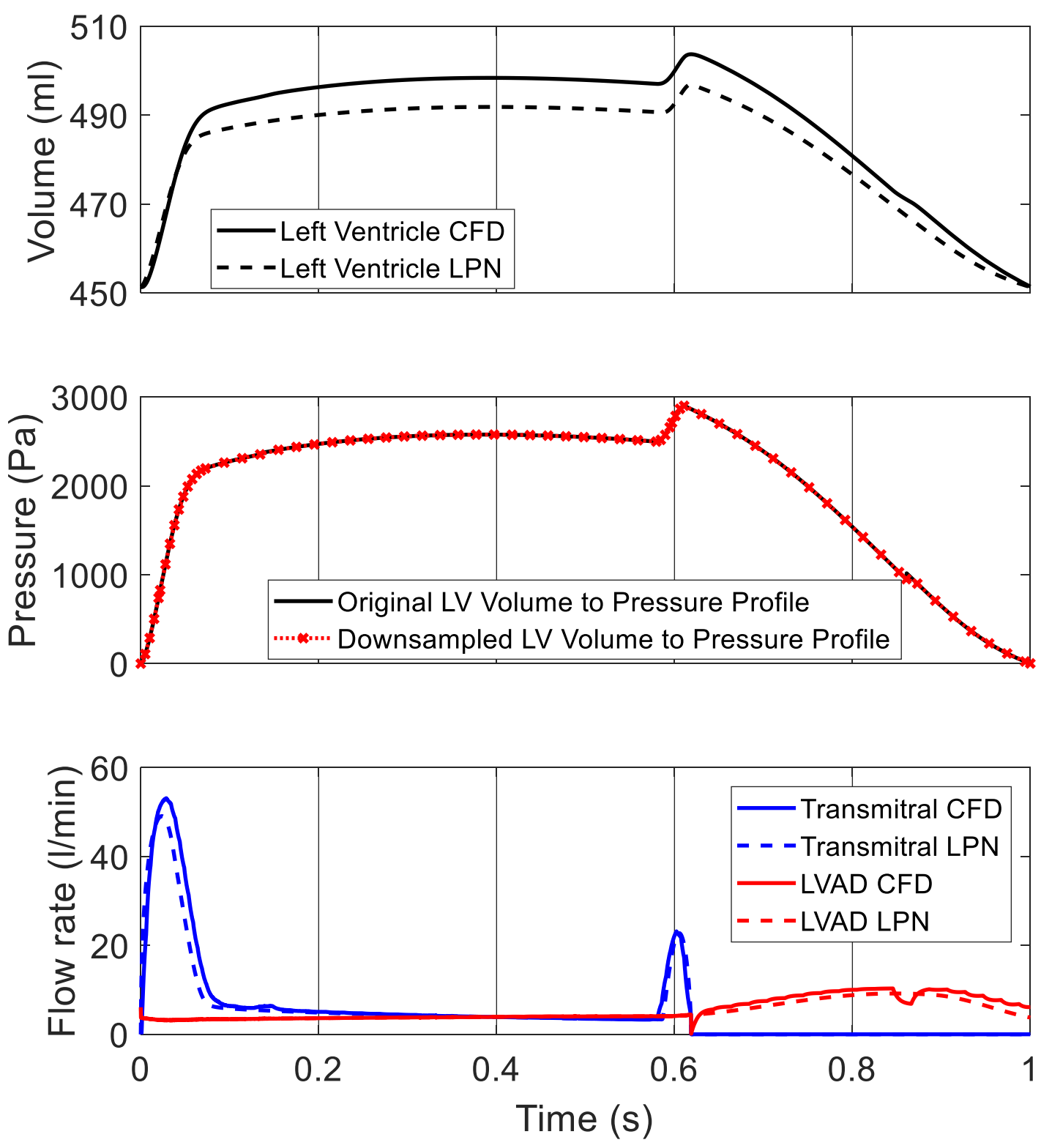

Journal of Biomechanics (Accepted: $1^{\text {st }}$ March 2018) 


\section{Figure 3}

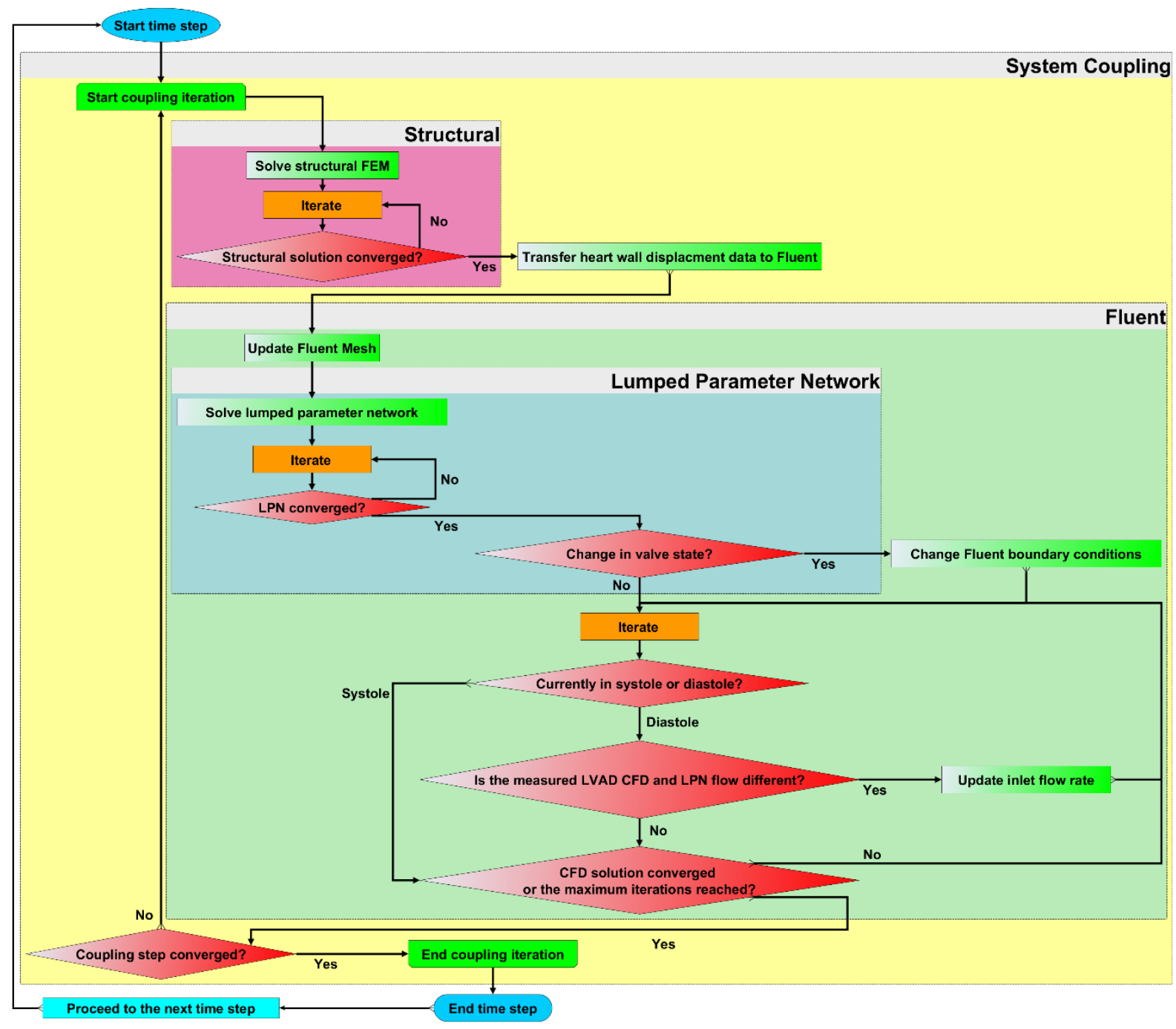


Figure 4

Journal of Biomechanics (Accepted: $1^{\text {st }}$ March 2018) 


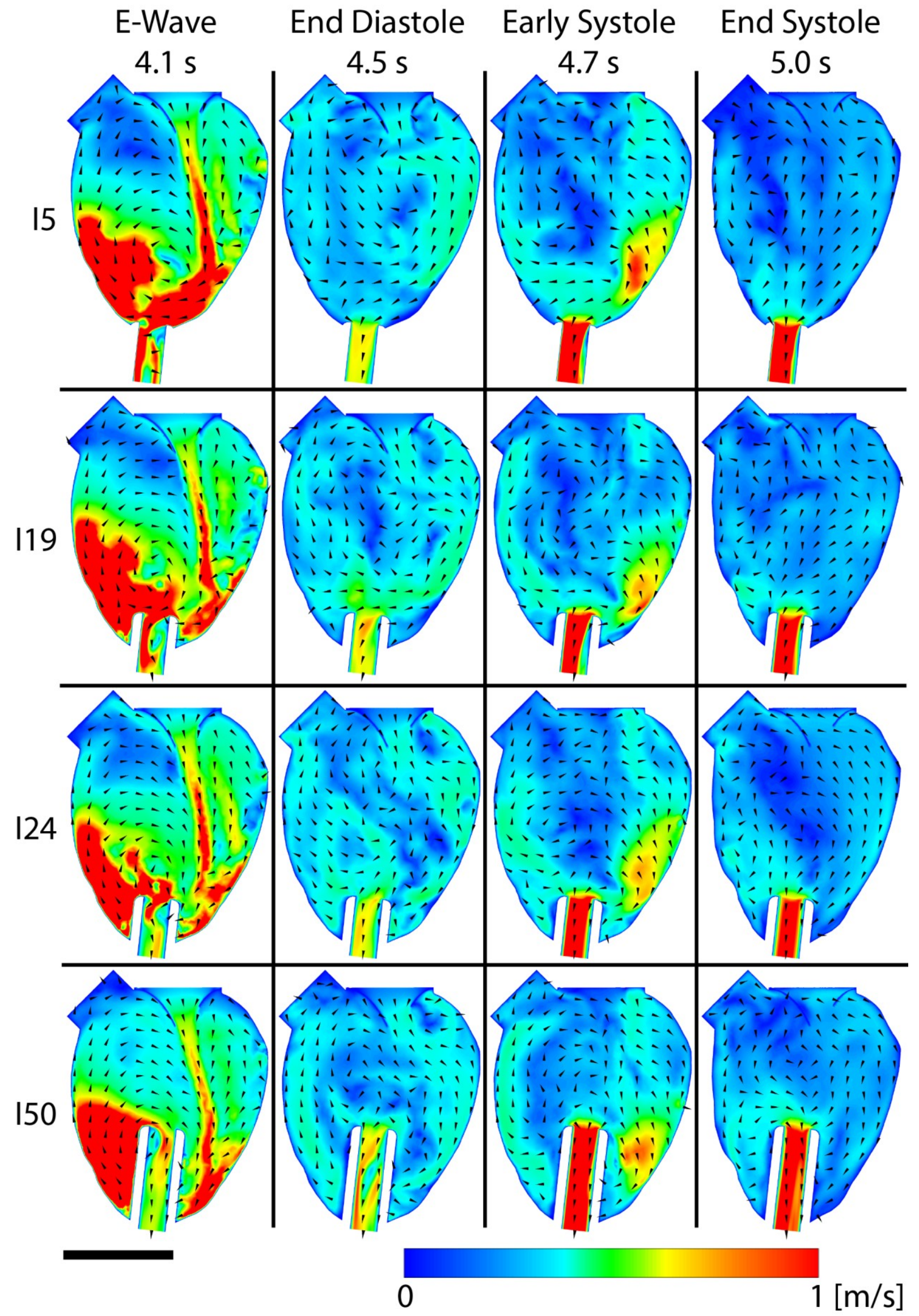

Journal of Biomechanics (Accepted: $1^{\text {st }}$ March 2018) 
Figure 5

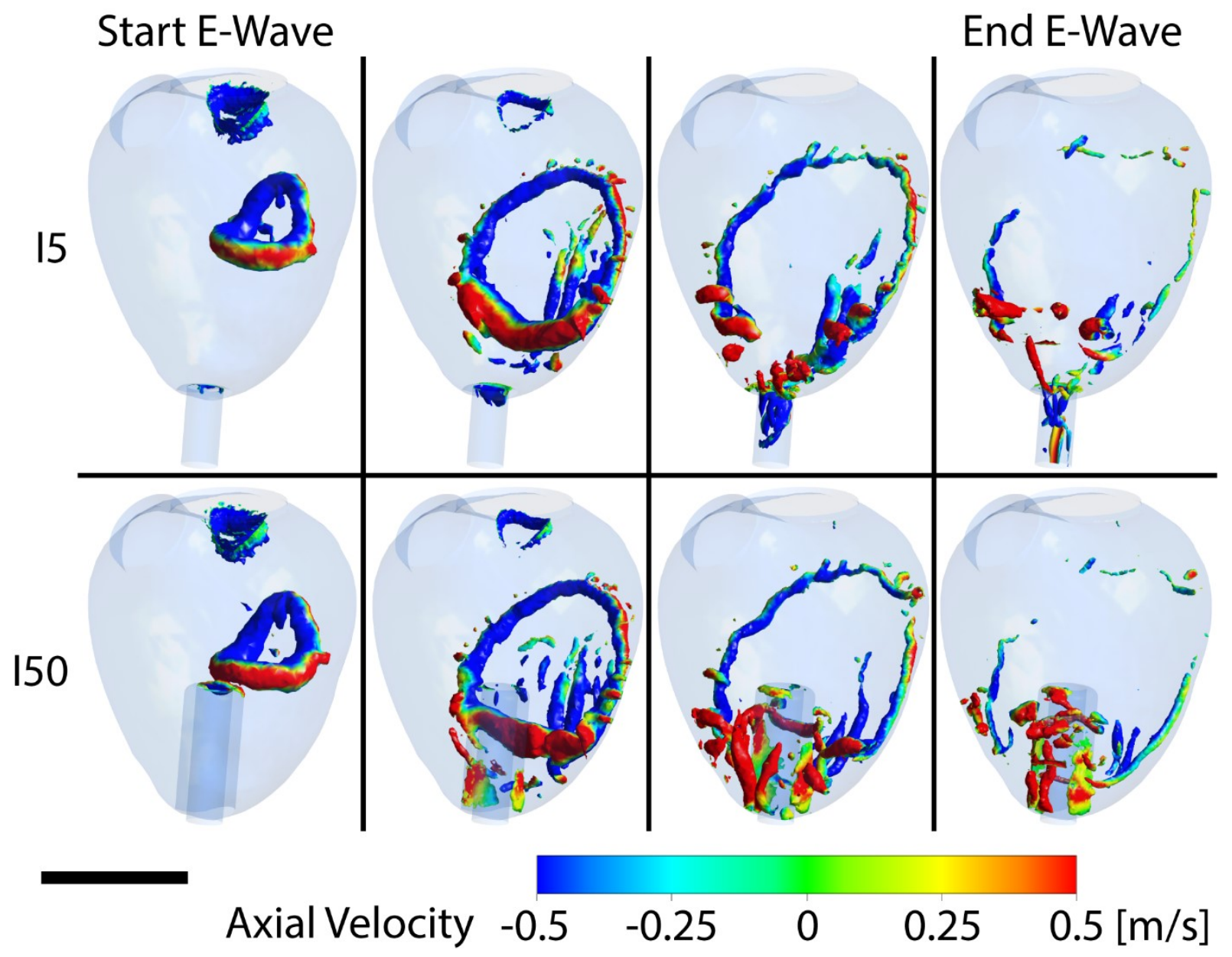

Journal of Biomechanics (Accepted: $1^{\text {st }}$ March 2018) 
Figure 6

Journal of Biomechanics (Accepted: $1^{\text {st }}$ March 2018) 


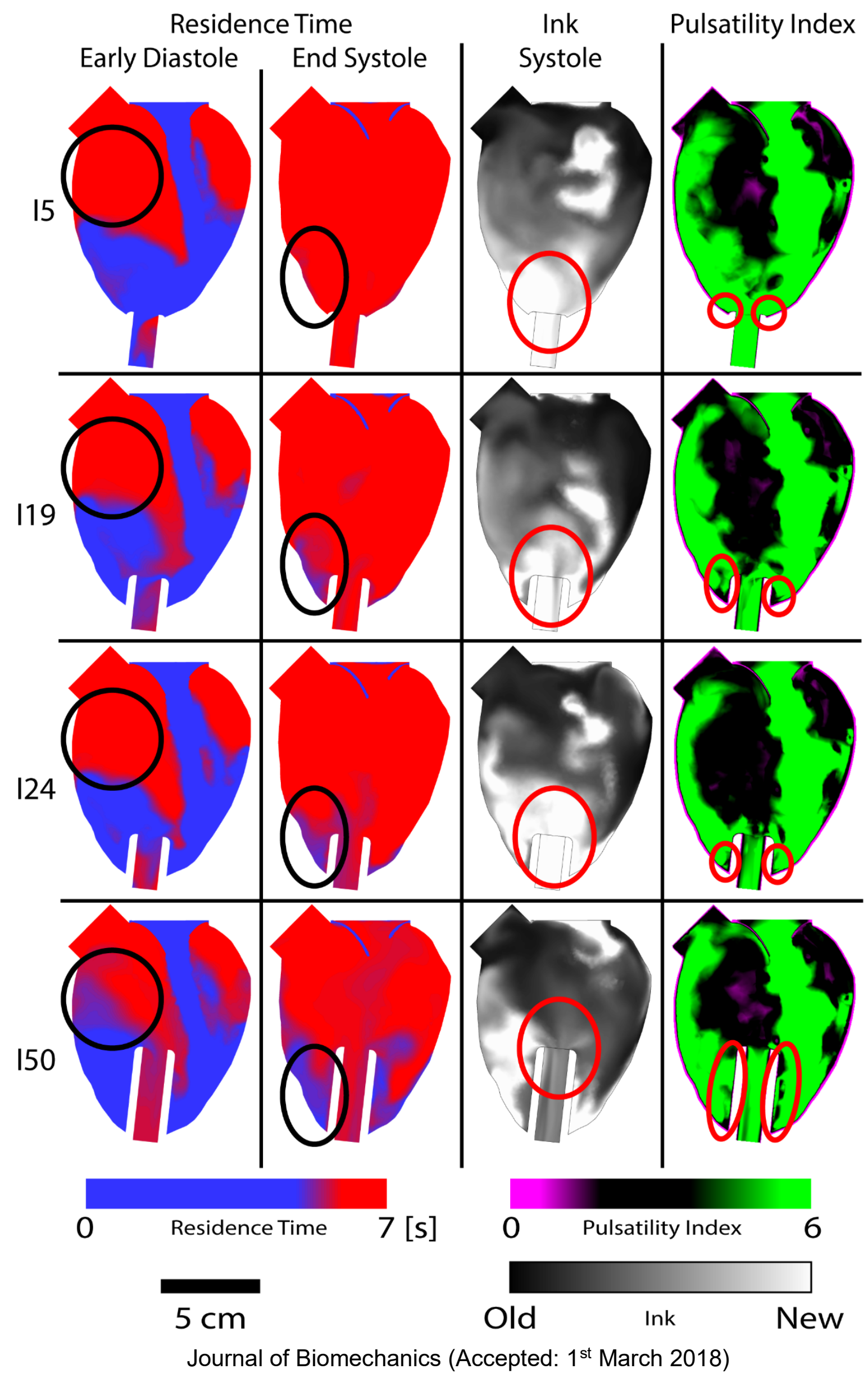


Figure 7

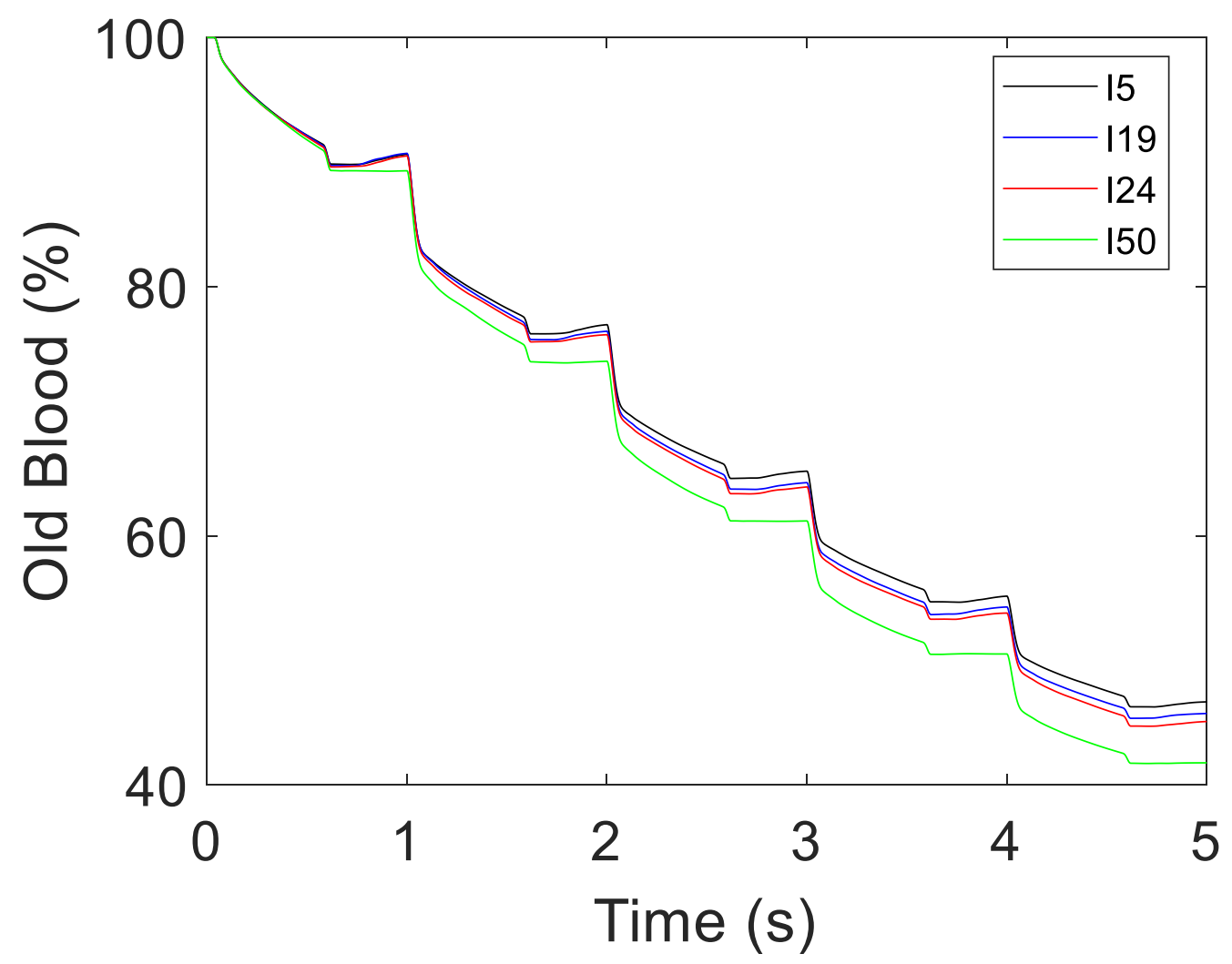

Journal of Biomechanics (Accepted: $1^{\text {st }}$ March 2018) 
Figure 8

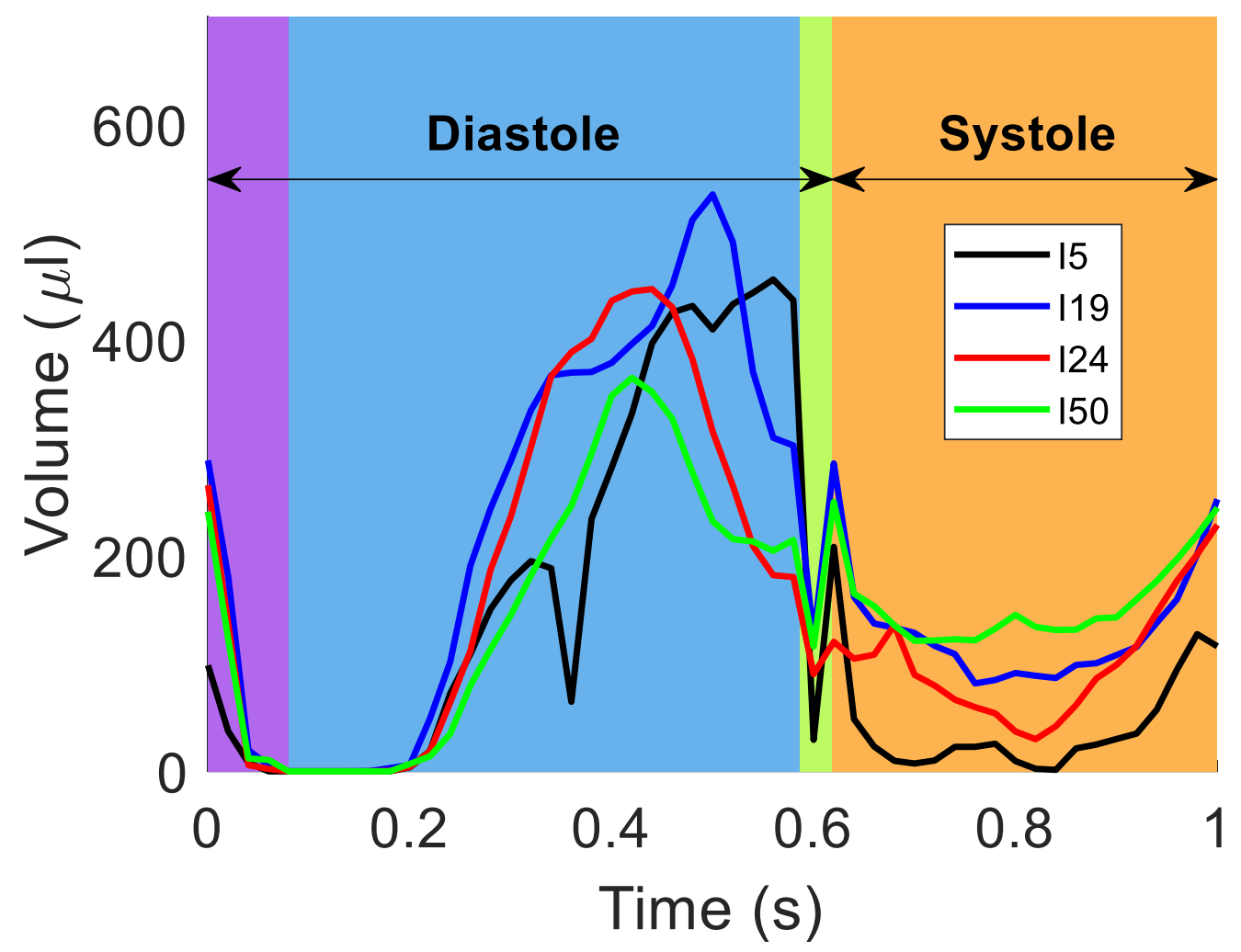

Journal of Biomechanics (Accepted: $1^{\text {st }}$ March 2018) 